\title{
Sitzung vom 10. Januar 1910.
}

Vorsitzender: Hr. H. Wichelhaus, Vizepräsident.

Der Vorsitzende eröflnet die Sitzung mit folgender Ansprache:

»Meine Herren! Bevor wir die wissenscbaftliche Tätigkeit des ueuen Jahres beginnen, liegt es mir ob, eine betrübende Mitteilung zu machen." $\mathrm{Hr}$. Geheimrat Prof. Dr.

\section{Carl SchotTen}

ist nach längerem Leiden gestern rerschieden. Er gehörte dem Vorstande der Gesellschaft seit 1895 ununterbrochen, teils als stellvertretender Schriftführer, teils als Schriftlübrer, an.

Am 12. Juli 1853 in Marburg geboren, promovierte Carl Schotten 1878 in Berlin und wurde alsbald Assistent bei A. W. Hofmann. 1881 ging er als Assistent an das physiologische Institut über, habilitierte sich 1883 als Privatdozent und wählte für seine Vorlesungen das Thema: „Chemie der Ernährung«.

Von seinen wissenschaftlichen Arbeiten sind besonders hervorzuheben: die Schotten-Baumannsche Benzoylierungs-Reaktion und die Aufspaltung des Piperidins, welche zur $\delta$-Amido-valeriansäure fübrte.

In der letzten Zeit war seine Tätigkeit wesentlich dem Kaiserlichen Patentamte gewidmet.

Ich ersuche Sie, sich zu Ebren des Verstorbenen von Ihren Sitzen $z u$ erheben.*

Der Vorsitzende teilt ferner mit, daß die in der letzten Generalrersammlung gewäblten Vorstandsmitglieder und Ehrenmitglieder die auf sie gefallene $W a b l$ angenommen baben. Seitens der neu ernannten Ehrenmitglieder sind an den Generalsekretär folgende Schreiben gerichtet worden:

Berichte d. D. Chem. Gesellschaft. Jahrg. XXXXII. 
Paris, 18 Décembre 1909.

Cher Monsieur,

J'ai bien reçu votre lettre en date de Décembre 1909 par laquelle vous m'informez que, dans sa séance du 15 Décembre dernier, la société chimique d'Allemagne m'a nommé nembre d'honneur de cette société. Je suis très sensible ì cet honneur et je rous prie de vouloir bien être l'interprite de tous nes remerciements auprès du bureau de votre société.

Je vous prie, Monsieur, de vouloir bien agreer l'expression de mes sentiments de baute considération.

H. Ise Chatelier, Membre de l'Institut.

Paris, le 25 Décembre 1909.

Monsieur et honoré Collègue,

Très souffrant depuis quelque temps je n'ai pu repondre aussitôt à votre lettre du 16 courant, dans laquelle vous voulez bien m'annoncer que, dans sa séance du 15 Décembre, la "Deutsche Chemische Gesellschaft m'a nommé Membre d'Honneur.

Veuillez, je vous prie, être auprès de nos collègues de la Sociéte, l'interprête de mes meilleurs sentiments de gratitude pour le grand honneur qu'ils m'ont fait et dont je seas tout le prix et agréer, Monsieur le Secrétaire général, l'assurance de ma considération la plus distinguée

A. Haller.

Milano, li 27. Dezember 1909.

Sebr geehrter Herr Kollege!

Ihr geehrtes Schreiben vom Dezember d. J., wonach die Generalrersammlung der Deutschen Chemischen Gesellschaft mich auf Vorschlag des zeitigen Ausschusses am 15. d. zum Ehrenmitglied erwählt bat, ist mir soeben zugegangen.

Die Erwählung ist für mich eine große Ebre und angenehme Überraschung, Ehre, die ich um so höher schätze, als sie völlig unverdient kommt, indem ich, seit dem Jahre 1863 beständig im Auslande lebend, kaum mehr eine Veröffentlichung in deutscher Sprache gemacbt habe.

Mit innigstem Danke nehme ich die Wabl selbstrerständlich an und ersuche Sie, hocbgeebrter Herr Kollege, denselben vor allem den Vorstandsmitgliedern auszusprechen. 
Seit verschjedenen Jahren mit einer Revisionsarbeit der allernächsten Benzolsubstitutionsprodukte beschäftigt, bezüglich deren nicht wenig recht ungenaue Angaben in die Literatur übergegangen sind, hoffe ich, in kürzester Zeit mich an den Arbeiten der Gesellschalt beteiligen zu können.

Mit hochachtungsvollem Gruße

Ihr ganz ergebener

W. Körnèr.

Vom Verband Deutscher Elektrotechniker ist die Anzeige eingegangen, daß er seine Hauptrersammlung rom 25.-28. Mai 1910 in Braunschweig abhalten wird.

Als außerordentliche Mitglieder sind aufgenommen:

Hr. Traun, H. O., Hamburg;

- Bergell, C., Kiel;

- Hesse, H., \;

* Levelace, Prof. Dr. B. I., Alabama;

D Engelbardt, A., Charlottenburg;

D van Name, Prof. R. G., New Haven;

- Baither, Dr. O., Griesheim;

"Bergmann, M., Berlin;

\ Johlin, J., > ;

- Wagner, A., ";

- Helferich, B., >

- Severin, Dr. J., ;

- Zemplèn, Dr. G., \;

\Scriba, C., Darmstadt;

» Sternberg, Prof. Dr. M., Wien;

- Fritzmann, E., St. Petersburg;

- Baume, Dr. G., Genf;

> Dehn, B. F., Jena;

- Haworth, W. N., Göttingen;

- Henjes, F.,

- Rantscheff, D., 》 ;

- Pauly, F.,
Frl. Fiedler, A., Gottingen;

Hr. Gughialmelli, Dr.J., Buenos Aires;

- Bödecker, E., Zürich;

- Haid, Dr.R., Klosterneuburg;

- Landau, B., Berlin;

- Ching-KoOu, ;

- Zsuffa, M., Cbarlottenburg;

- Beutner, Dr. R., Karlsrube;

- Bratring, K., Berlin;

- Weibezahn, K., * ;

- Smolnikoff, J., " ;

- Lichtenstadt, I., \;

- Pinner, Dr. E.L., Charlottenburg;

- Buchner, Dr. K., Charlottenburg;

- Caland, Dr. P., Amsterdam;

- Ritter, H., Karlsruhe;

* Bergen, J.ron, \;

-Fabricius, A.von" ;

-Jacobsohn, Dr. W., SteglitzDahlem;

- Terres, E., Östrich;

- Mosebach, G., Berlin;

-Reinbeck, M., Charlottenburg; 
Hr. Hild esheimer, Dr.A.,Berlin;

\Schlenk, Dr. W., München;

* Mair, L., München;

\Hirsch, P., \;

» Steuer, Dr. O. R., Mainkur;

- Birstein, Dr. G., München;

- Buchbinder, W., Dresden;

"Ellinger, Ph., Greifswald;

- Renschler, E., Stuttgart;
Hr. Schmidtmann, Dr. H. Aschersleben;

》 Teichmann, Dr. H., Rauxel,

- Russ, K., Tübingen;

- Binder, K., > ;

》 Beck, Chr., \;

》Spitz, W., Lankwitz;

\Scheffer, Dr. W., Wilmersdorf.

Als außerordentliche Mitglieder werden rorgeschlagen die HHrn:

Meyer, Dr. Ed., Sydowsaue (durch Graf H. Luxburg und F. Schneider);

Göddertz, Schlüterstr. 55, Charlottenburg (durch F. Mylius und B. Lepsius);

Wilenski, Michael, Zieglerstr. 66, Bern (durch V. Kohlschütter und F. Ephraim);

Paulsen, Edgar, Dänischestr. 43, Kiel (durch C. Harries Behrens, Otto, Waitzstr. $26 \mathrm{II}$,$\} und 0 . \mathrm{Mumm}$ );

Knopf, Martin, Niebuhrstr. 71, Charlottenburg (durch R. Pschorr und D. Holde);

Grether, Dr. Ernst, Gesellschaft für chemische Industrie, Leopoldshōhe-Baden (durch E. Preiswerk und B. Schreiber);

Glahn, Dr. Ph., Möhringstr. 9, Wiesbaden (durch I. Oppermann und K. Albrecht);

Braß, Dr. Kurt, Kreuzlinstr. 1 III, München (durch G. Schultz und H. Jacob);

Perl, Dr. Ing. Alfred, 67 Claremont Rd. Moss Side, Manchester SW. (durch E. Knecht und J. H. Reynolds); Goldschmidt, Stephan, Liebherrstr. 4 II, München (durch O. Dimroth und H. Wieland);

West, Clarence J., 815 So. State Str., Ann Arbor, Mich. (durch H. H. Willard und L. H. Cone);

Vries, Dr. Otto de, Departement ran Landbouw, Buitenzorg (Java) (durch A. P. N. Franchimout und H. J. Backer);

Gutbier, Prof. Dr. A., Bismarckstr., Er-

$\begin{aligned} & \text { langen } \\ & \text { Broek, Dr. J. A. van den, Pippinstr. 30, } \\ & \text { Gauting (Holl.) }\end{aligned}$
$\begin{gathered}\text { Müller, Dr. D., Daheimstr. 1, Dresden- } \\ \text { Plauen }\end{gathered}$


Steinhorst, Hermann, Sigismundstr. 4, Berlin W. 10 (durch O. Wallach und A. Kötz);

Weil, Dr. Stanislaus, Marszalkowska 14, Warschau (durch

St. von Kostanecki und V. Lampe);

Full, Prof. Dr. Camil, Kgl. Realschule, Würzburg,

Leone, Dr. T., Universität, Palermo, $\left\{\begin{array}{l}\text { (durch J.F.Holtz } \\ \text { und R. Daum); }\end{array}\right.$

Reuter, Dr. B., Farbwerke, Höchst a. M. (durch Fr. Stolz und G. Korndörfer);

Kelley, G. L., 272 Main St., Everett, (durch Mass., U. S. A.

C. L. Jackson

Bigelow, H. E., 12 Oxford Str., Camund bridge, Mass., U. S. A.

A. H. Fiske);

Stoakley, I. J., Chemical Laboratory, Cambridge (Engl.)

(durch W. J. Pope und S. Ruhemann);

Simonsen, Jobn Lionel, The Presidency College, Madras

(Indien) (durch A. Lapworth und W. H. Perkin);

Dohrn, Dr. Reinhardt, Zoologische Station, Neapel (durch R. Pschorr und L. Jablonski);

Radulescu, Don, Leibnizstr. 27 I, Charlottenburg (durch H. Leuchs und F. Sachs);

Averbeck, Heinrich, Schillerstr. 1, Jena (durch L. Knorr und P. Rabe);

Emde, Privatdozent Dr. Hermann, Geysostr. 21, Braunschweig (durch H. Beckurts und J. Tröger);

Richter, Dr. Georg, Wilhelmstr. 7, Oranienburg (durch J. Rawitzer und P. Jacobson);

Henkel, Dr. Hugo, per Adr. Henkel \& Co., Chemische Fabrik, Düsseldorf (durch P. Jacobson und R. Stelzner); Nolte, Ernst, Herderstr. 7, Jena (durch L. Wolff und O. Rasch).

Für die Bibliothek sind als Geschenke eingegangen:

26. Fehling, H. v. Nenes Handwörterbuch der Chemie, fortgesetzt von C. Hell und C. Haussermann. 108. Lieferung. Brannschweig 1909.

106. Jahresbericht über die Fortschritte der Chemie und verwandter Teile anderer Wissenschaften, begründot von J. Liebig und H. Kopp, herausgegeben von J. Trōger and E. Baur. Für 19051908, Helt 3 und 4. Braunschweig 1909.

1880. Gmelin-Krauts Handbuch deranorganischen Chemie. 7. Auflage, herausgegeben von C. Friedheim, fortgesetzt von F. Peters. 110.-116. Lieferung. Braunschweig 1909. 
561. Holıle, D. Untersuchung der Mineralöle und Fettc, sowie der ihnen verwandten Stoffe. 3. Auflage. Berlin 1909.

568. Partheil, A. Kurzgefaßtes Lehrbuch der Chemie für Mediziner und Pharmazcuten. Organischer Teil. Abt. I: Die Chemio der Fettkōrper. Bonn 1909.

569. Osborne, Th. B. The regetable proteins. London 1909.

580. Gerlach, V. Physiologische Wirkungen der Benzoesäure und des benzoesauren Natrons. Wiesbaden 1909.

608. Ausschuß für das Duisberg-Jabiläum. Die Jubilăumsfestlichkeiten zil Ehren dus Hrn. C. Duisberg. Leverkusen 1909.

68i. Battle, H. B., and Gasconne, W. J. Chemical conversion tables. Baltimore 1909.

807. Biedermann, R. Die Sprengstoffe, ihre Chemie und Technologie. Leipzig 1910.

808. Goldberg, J., und Friedmann, H. Die Sulfosăuren des Antbrachinons und seiner Derivate. Berlin 1909.

Der Vorsitzende:

II. Wichelhaus.
Der Schriftführer:

A. Bannow.

\section{Mitteilungen.}

\section{Bericht der Internationalen Atomgewichts-Kommission firr 1910.}

Mitglieder: F. W. Clarke, W. Ostwald, T. E. Thorpe, G. Urbain.

(Eingegangen am 8. November 1909.)

Seit der Erstattung unseres letzten Berichtes bat eine lebhafte Tätigkeit bezüglich der Atomgewichtsbestimmungen stattgefunden. Nachstehend ist eine kurze Übersicht der Ergebnisse gegeben.

Ohlor. Eine neue Vergleichung zwischen Chlor und Sauerstoff ist durch Guye und Fluss ') ausgefübrt worden. Nitrosylchlorid, NOCl, wurde zuerst gewogen, über Silber geleitet, welches das Chlor auinahm, sodann über erhitztes Kupfer zur Aufnahme des SSauerstoffes und zuletzt über metallisches Calcium, welcbes den Stickstoff zurückhielt. Auf solche Weise wurde die vollständige Analyse dieser Verbindung durchgefübrt. Aus dem Verhältnis des Sauerstoffes zum Chlor folgt $\mathrm{Cl}=35.468$.

1) Journ. chim. phys. 6, 732. 